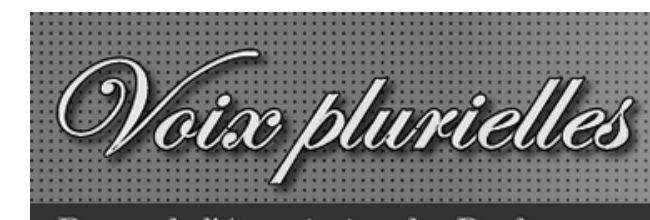

Revue de l'Association des Professeur-e-s de Français des Universités et Collèges Canadiens

Voix plurielles

Volume 4, Numéro 2 : septembre 2007

Mylène Tremblay

\title{
L'indécence autobiographique chez Nelly Arcan et Marie-Sissi Labrèche ou comment ravir l'attention (la tension) du lecteur
}

Citation MLA : Tremblay, Mylène. «L'indécence autobiographique chez Nelly Arcan et Marie-Sissi Labrèche ou comment ravir l'attention (la tension) du lecteur ». Voix plurielles 4.2 (septembre 2007). 


\title{
L’indécence autobiographique chez Nelly Arcan et Marie-Sissi Labrèche ou comment ravir l'attention (la tension) du lecteur
}

\author{
Mylène Tremblay \\ Collège François-Xavier-Garneau / Université Laval
}

Mai 2007

L'indécence, par son attaque en règle de la pudeur, renvoie spontanément à la sexualité. Avec sa découverte de la psychanalyse, Freud a bien fait ressortir l'importance de la sexualité dans la civilisation occidentale. En effet, l'objet de prédilection du refoulement, la cause des grandes hystéries, résidait d'abord dans les pulsions sexuelles. Toutefois, dans Au-delà du principe de plaisir, Freud identifie une pulsion qui irait au-delà de la notion de satisfaction, la pulsion de mort, que Lacan appellera jouissance. Dans cet article, je ne souhaite pas limiter l'indécence à la question sexuelle, mais je la rattacherai à la question de l'obscène, telle que l'a développée le psychanalyste Willy Apollon du GIFRIC (Groupe interdisciplinaire freudien de recherche et d'intervention cliniques) à Québec: selon le chercheur, la culture «délimite les figures de l'obscène où la jouissance accomplit son travail de mort, qu'elle oppose au croyable qui conditionne la coexistence et la vie commune ${ }^{1} \gg$. L'obscène se constitue donc de ce qui est exclu de la scène sociale, de ce à quoi une société n'adhère pas à un moment donné, un hors-scène.

Chez certains individus, le croyable ne suffit pas à canaliser la pulsion. Plusieurs auteures, par exemple, dénoncent l'étroitesse de leur propre société ou de leur milieu familial qui sont impuissants à les sortir de l'emprise de la jouissance. Elles refusent la fausse pudeur, qu'elles jugent artificielle et aliénante. Elles abordent l'obscène pour ne pas être absorbées par lui, détruites par lui. Elles deviennent indécentes, comme en témoigne un passage de La Lune dans un HLM :

Et là j'aurai envie de hurler comme une manifestante d'aller te faire voir loin loin, maudite folle, que je ne suis certainement pas ta fille, que tu as dû me kidnapper à la pouponnière quand je suis née, que ça ne se peut pas que je sois sortie de toi comme ton bout d'intestin! Mais ça ne se dit pas. On ne dit pas de telles choses à sa mère, surtout si elle est malade, si elle n'est pas armée pour la vie. Pour quelle fille ingrate passe-t-on? Alors je l'écris. Je n'ai pas le choix. C'est ça ou c'est comme si je me tirais une balle dans la tête, ou plutôt comme si je te laissais m'avaler, me manger toute crue. Lustucru ${ }^{2}$. 
L'eusses-tu cru? La formule enfantine rejoint la préoccupation première de toute éducation : transmettre d'abord la foi en la vie, ensuite, la foi en la vie sociale. Mais il faut être crue pour pouvoir croire. Quand le doute s'installe de part et d'autre, quand la foi n'est pas transmise, que reste-t-il, en dehors de l'autodestruction? La psychanalyse, comme l'esthétique d'ailleurs, devient un lieu possible pour l'obscène.

Nombreuses sont les œuvres essentielles qui surgissent d'un silence imposé par l'espace social. Mais la revendication de l'obscène par une subjectivité énonciatrice semble être la marque de notre temps, l'autofiction. On évoque l'horreur, l'insupportable, le Réel, on invoque la nécessité d'en parler et on le fait en revendiquant une expérience dans la réalité. Trois aspects seront retenus pour étudier ces stratégies singulières d'écriture : l'ambiguïté de l'énonciation, la structure et l'ambivalence de la position subjective.

\section{L’énonciation}

Plusieurs fois, en entrevue, Nelly Arcan a dit regretter s'être jetée en pâture aux interviewers d'émissions télévisuelles populaires, de s'être trop livrée. Elle s'est même promise d'écrire une prochaine œuvre à la troisième personne pour « savoir si [elle] était capable d'écrire autre chose que sa propre vie, même si en écrivant [sa] vie il y a beaucoup de fiction qui traverse le texte ${ }^{3} »$. Dès la sortie de Putain, Nelly Arcan a toujours reconnu s'être servie de l'écriture pour exorciser son passé d'escorte. Dans Folle, l'autofiction continue puisqu'elle fait référence à ses rapports difficiles avec les médias, son passage chez Christiane Charrette ${ }^{4}$ où son amant l'aurait vue pour la première fois. La narratrice se présente d'ailleurs comme «l'auteur d'un livre titré Putain ${ }^{5}$ ». Toutefois, il ne s'agit pas d'une autobiographie parce que la narratrice n'établit pas une association explicite avec l'auteure. Le titre porte même la mention roman. Le contenu reste intime et s'apparente au journal intime, mais le livre ne se présente pas ainsi explicitement. Les destinataires avoués, ses parents, son psychanalyste, ses clients, les femmes, un vous anonyme, donnent l'illusion de visées à la fois personnelles et littéraires dans l'écriture. L'ambiguïté est voulue, soutenue, maintenue. Le lecteur est interpelé comme complice, comme voyeur et comme confident.

Dans les ouvrages de Marie-Sissi Labrèche, l'auteure prend plaisir à jouer avec son nom. Le nom de la protagoniste s'apparente en effet étrangement avec le nom de l'auteure: Sissi Labrèche (plutôt que Marie-Sissi Labrèche). Le deuxième ouvrage continue les jeux onomastiques en reprenant cette fois-ci le nom de l'auteure dans le titre ${ }^{6}$. La narratrice s'appelle cette fois-ci «Émilie-Kiki ${ }^{7}$ », mais elle partage avec la protagoniste bien des caractéristiques. D'abord, sa brèche et elle font la paire ${ }^{8}$. Ensuite, le portrait familial reste plus ou moins le même, avec la mère folle, morte et la grand-mère autoritaire. Enfin, plusieurs passages de Borderline trouvent écho dans La Brèche.

Borderline : «T'es bonne en crisse pour raconter des histoires. T'es bonne en crisse pour raconter des histoires qui rendent ta mère folle ${ }^{9} \gg$.

La Brèche : «ÉMILIE-KIKI, TU RENDS FOLLE TA MÈRE!

C'EST DE TA FAUTE SI ON DOIT TOUT LE TEMPS L'INTERNER $!^{10} »$ 
Le projet d'écriture de La Brèche s'inscrit explicitement dans un programme d'autofiction... aux dires de la protagoniste. La narratrice a donc des projets d'écriture. La première phrase du roman résume très efficacement l'intrigue: « J'ai vingt-six ans et je baise avec mon prof de littérature. Fuck the system do it, do it, do it, do it yeah! ${ }^{11}$ » La première phrase marque son triomphe, parce qu'elle a déployé bien des efforts pour convaincre le professeur en question. Mais là ne s'arrêtent pas ses désirs :

[...] avant qu'il insère sa queue dans ma brèche, je lui avais dit que j'avais envie d'écrire notre histoire, que j'avais envie de me transformer en écrivain de la pire espèce, en cannibale de l'âme, en timbrée qui s'amuse à mélanger la réalité à la fiction au point de ne plus savoir ce qui est vrai et ce qui est faux, ce qui est bien et ce qui est $\mathrm{mal}^{12}$.

Le troisième roman de Marie-Sissi Labrèche reprend ce jeu de brouillage dans la situation de communication entre un auteur et son lecteur. Alors que le roman est commencé, croit-on, et que la Première lettre raconte le sujet du roman ( «C'est 1'histoire de Léa et de sa mère folle. ${ }^{13}$ »), la narratrice-auteure nous renvoie tout de go à l'exergue: «Oui, maman, c'est à toi que je m'adresse, c'est à toi que je dédie ce livre, le Elle en exergue, c'est le tien ${ }^{14} »$. Comme dans les livres précédents, sa mère est une «vraie folle ${ }^{15}$ ", une "folle à $\operatorname{lier}^{16}$ » diagnostiquée, et menacée d'internement.

\section{La structure}

La dissolution d'une ligne de partage claire entre le réel et la fiction entraîne le lecteur derrière le voile de la pudeur, dans les bas fonds de l'intime ou les tréfonds de l'âme. Avec le risque de réveiller en lui-même ses propres angoisses, ses pulsions refoulées. Labrèche et Arcan, dans leur écriture, semblent puiser à même le ça, ce « réservoir de pulsions ", ce « dépotoir de tension ». L'ambiguïté de l'énonciation devient une stratégie fort utile, voire nécessaire pour entraîner le lecteur vers les sables mouvants de la jouissance, un terrain glissant qu'il aurait évité à tout prix, en temps normal, cet hypocrite lecteur. L'indécence autobiographique, au sens strict de dévoilement impudique, devient un leurre pour aborder des sujets plus morbides et horrifiants, comme la jouissance féminine. La structure des romans est à l'image de cette plongée dans l'obscène. Je ne m'attarderai ici qu'aux premiers romans des deux auteures.

Les romans Borderline et Putain rappellent les fondements mêmes de la psychanalyse : la cure par la parole. Ainsi, le prologue de Borderline s'ouvre sur des souvenirs et l'épilogue se termine chez le «psy ». L'enchaînement des chapitres plonge la protagoniste dans des souvenirs de plus en plus anciens... jusqu'à la scène du miroir fracassé, qui rappelle les enjeux mortifères de l'image de soi et du corps morcelé. Sissi ressent d'ailleurs une envie irrésistible de plonger dans l'eau du fleuve pour rejoindre les étoiles et la grand-mère. L'œuvre de Labrèche contient également des termes de psychologie et tout un vocabulaire psychiatrique qui permettent le parallèle avec la démarche thérapeutique.

L'organisation textuelle de Putain produit un effet similaire, mais le simulacre est encore plus près de la représentation d'une cure. Il ne s'agit plus seulement d'établir des liens entre des 
événements présents et des traumatismes passés, mais de mettre en scène la parole pleine dans un flot de discours ininterrompu, à bâtons rompus. L'association libre est la "règle » qui prédomine : "Voilà pourquoi ce livre est tout entier construit par associations, d'où le ressassement et l'absence de progression, d'où sa dimension scandaleusement intime ${ }^{17} \gg$. L'effet de réel est percutant : «[...] je n'ai pas rêvé, [...] je n'ai rien inventé ${ }^{18}$ ». Le tourment de la narratrice est palpable. Il ne s'agit pas d'une histoire, mais d'une pensée à l'état pur qui cherche à se dire au fil des mots. Le seul exercice qu'elle se permet est la synthèse. Elle ne peut tout dire de sa vie de putain, "l'écriture est un principe de mort ${ }^{19}$ ", dira-t-elle; on y verrait presque l'écho lacanien de la célèbre formule « le mot est le meurtre de la chose ». Il lui faut réduire ${ }^{20}$.

Malgré la réduction, les effets de répétition sont saisissants de vérité alors que le récit en arrive à progresser au rythme des confessions, des révoltes, des régressions : «[...] voilà pourquoi nous en revenons toujours à ça, au travail de la séduction dans le récit de mon malheur, à la façon que j'ai de haleter mon histoire comme si j'étais en plein accouplement, ma façon de languir et de laisser languir $[\ldots]^{21} \gg$. Comme une véritable analysante qui se complait dans sa plainte et n'arrive qu'avec peine à révéler ce qu'elle sait depuis toujours, la narratrice se dénude petit à petit en se servant des anecdotes de putasserie pour ralentir le rythme du véritable obscène. On sent une forme de jouissance dans cette écriture parfois hypnotisante.

Putain est une authentique recherche de sens. Dans le livre, il y a quatre-vingts occurrences du mot " pourquoi ». La narratrice ressasse les mêmes faits, les mêmes souvenirs. La vie d'une putain est à l'image même de la répétition. Le mot « encore » revient quatre-vingt-quinze fois... et ce n'est pas pour réclamer du plaisir, loin de là. À plusieurs reprises, les redites sont mises en évidence : « je l'ai déjà dit je crois $^{22}$ »; « de toute façon il n'y a rien à faire et je l'ai déjà dit ${ }^{23}$ »; « ça aussi je l'ai déjà dit ${ }^{24}$ ». La narratrice n'a pas peur de répéter, elle se risque à le faire :

[...] je parle comme j'écris [...], je m'adresse à ce qui se tient ici en sachant que ça ne sert à rien, qu'à parler sans arrêt, ça ne sert à rien mais il faut s'entêter pour ne pas mourir sur le coup d'un silence trop subi, tout dire plusieurs fois de suite et surtout ne pas avoir peur de se répéter, deux ou trois idées suffisent pour remplir une seule tête, pour orienter toute une vie ${ }^{25}$.

L'exercice rappelle le but ultime de toute psychanalyse, la découverte, l'analyse et le désamorçage du fantasme, cette petite formule du désir, qui circonscrit la jouissance à l'origine de la répétition.

Pour la narratrice, le récit idéal serait celui du psychanalyste qui pourrait faire de son histoire une histoire de $\operatorname{cas}^{26}$, réduire son mal être à une cause précise. Elle se plaît à imaginer ce récit, mais elle se sent impuissante à le produire : «[...] mais ce n'est pas de ça que je parle, ça n'a jamais été de ça, ce dont je parle, ce n'est pas à moi de vous le dire, je le répète sans pouvoir mettre le doigt dessus [... $]^{27} » ;$ « [...] je suis malade de ne pas pouvoir nommer le mal que j'ai $[\ldots]^{28} \gg$. Putain rappelle à maints égards Les Mots pour le dire de Marie Cardinal sans la fin libératrice. Sans doute est-ce cette impasse qui a fait dire à plusieurs que le roman se déployait autour d'une structure circulaire. 


\section{La position subjective}

Après l'analyse d'une ambiguïté énonciative qui fait miroiter aux lecteurs des révélationschoc sur l'intimité la plus indécente, après la mise au jour d'une structure propice à la révélation subjective, je m'intéresserai en dernier lieu à la représentation d'une position particulière vis-àvis du savoir révélé. C'est au cœur même de l'ambivalence que je situe la tension de la lecture, le malaise que plusieurs lecteurs prennent plaisir à révéler sur internet. J'étudierai moins les vérités toutes nues que les narratrices crachent au visage ou aux yeux du lecteur dans l'œuvre, que la position choisie par ces mêmes narratrices face à leur savoir.

Dans Putain, Nelly Arcan fait une dénonciation en règle du système patriarcal. Elle critique avec véhémence la pression sociale exercée sur le corps féminin ${ }^{29}$. Comme une victime, elle se sent offerte aux regards des hommes et captive de cette position. On ne semble lui avoir laissé le choix qu'entre la larve et la schtroumphette, autrement dit l'objet délaissé (la mère abandonnée) ou l'objet convoité (la petite fille choyée par le père) :

[...] c'est lui [mon père] qui a fait de moi une schtroumpfette, qui m'a élevée minuscule et bleue au milieu de grands champignons blancs, de forêts immenses habitées par les fées et les sorcières, c'est lui qui a choisi mon destin d'infirme attablée à ses pots de crème et à ses régimes [...], ce qui compte aujourd'hui est de rester petite le plus longtemps possible ${ }^{30}$.

Le père en effet, obnubilé par la religion, aux dires de la narratrice, ressassait constamment l'idée du péché et des dangers de la séduction, à tel point que la narratrice a eu l'impression de perdre son statut d'enfant chérie à l'âge de dix ans, l'âge où elle a cessé de dormir entre son père et sa mère, dans le lit conjugal. Quant à la mère, couchée dans un lit, elle se plaignait de l'absence et de l'indifférence du père que la jeune fille imaginait dans les bras des putains ${ }^{31}$. La narratrice, qui a pris le nom de sa sœur morte avant elle, comme pseudonyme de prostituée, Cynthia, semble prise dans cette histoire de couple et de famille sordide.

Toutefois, la jeune femme souhaite devenir maîtresse de sa destinée. Elle se fait putain et part en guerre contre certaines vérités insupportables : l'échec du couple, la convoitise des pères pour leur fille, le refus de la vieillesse et de la mort et, plus que tout, l'asservissement de la femme à son image. Tout y passe: l'anorexie, la chirurgie plastique, les cosmétiques, la féroce compétition féminine :

[...] si je ne sais pas crier ni gesticuler en dehors du lit, en dehors de la demande, alors peut-être des mots, ces mots pleins de mon cri qui pourront les frapper tous, et plus encore, le monde entier, les femmes aussi, car dans ma putasserie c'est toute l'humanité que je répudie, mon père, ma mère et mes enfants si j'en avais ${ }^{32}$.

C'est toute une vengeance qu'elle machine : contre ses parents, contre les clients, contre le lecteur même qu'elle dit vouloir scandaliser ${ }^{33}$. Les propos crus et vulgaires en témoignent; l'héroïne écrit comme elle putasse : 
[...] j'ai voulu [...] écrire ce que j'avais tu si fort, dire enfin ce qui se cachait derrière l'exigence de séduire qui ne voulait pas me lâcher et qui m'a jetée dans l'excès de la prostitution, exigence d'être ce qui est attendu par l'autre, et si le besoin de plaire l'emporte toujours lorsque j'écris, c'est qu'il faut bien revêtir de mots ce qui se tient là-derrière et que quelques mots suffisent pour être lus par les autres, pour n'être pas les bons mots ${ }^{34}$.

Toute l'ambivalence tient dans le néologisme créé pour désigner la position : la putasserie, la putasse rit... Le roman produit en effet une position subjective ambivalente, qui oscille entre la souffrance et la provocation, le savoir et le non-savoir, entre la lucidité et le déni. Selon Isabelle Boisclair, la figure de la putain tient du paradoxe puisqu'elle occupe tour à tour les positions de sujet et d'objet ${ }^{35}$. D'abord, malgré la haine du client, elle avoue éprouver du " plaisir $^{36}$ », souvent avec les trois premiers clients ${ }^{37}$. Elle apprécie l'argent ${ }^{38}$. Elle se targue d'être « la star de l'agence $^{39} »$; quand elle était petite, elle « étai[t] la plus belle ${ }^{40} »$; elle « enrage de ne pas être au palmarès des plus belles femmes du cinéma américain ${ }^{41} »$; lorsque les clients exigent une autre femme, elle avoue choisir la plus vieille et la plus moche pour se mettre en valeur ${ }^{42}$, tout en sachant qu'elle n'y est pour rien dans ce plaisir du client avec une putain ${ }^{43}$. Elle en a contre la servitude de la femme à son image, mais elle est irrésistiblement sous son emprise : elle rêve d'un royaume où elle est « la plus belle et la plus désirée de tous les royaumes ${ }^{44}$ », elle a besoin de se constituer par le regard de l'autre ${ }^{45}$. Elle reproche aux clients d'être assez vieux pour être son père, mais elle est littéralement obsédée par l'idée de l'inceste et de la «queue de son père $^{46}$ ", qu'elle souhaiterait retrouver avec son psychanalyste, le seul homme qu'elle aime. Elle apporte parfois des nuances ${ }^{47}$, mais elle tient beaucoup à sa maladie et c'est ce qui fait du roman une véritable plongée dans la jouissance féminine :

[...] tous les jours de jeunes malades s'éprennent de leur psychanalyste, ça fait partie du cours normal de la cure, je suis désolée mais je n'aime pas employer le terme analysante pour désigner les femmes suicidaires qui se prostituent, je préfère dire qu'elles sont malades, c'est plus honnête et plus excitant aussi, être malade $[\ldots]^{48} \gg$.

En fait, à la fin du livre, elle finira par révéler que ses parents avaient des relations sexuelles, qu'on l'excluait parfois du lit conjugal, mais l'interdit n'a pas suffi et n'a pas empêché qu'elle se livre elle-même comme petite fille à la convoitise des pères, la putain imaginaire du père :

[...] mais maman pourquoi est-ce que je ne peux pas voir papa tout nu et pourquoi lui peut-il te voir toute nue, voilà des questions auxquelles je n'ai jamais eu de réponse, enfin on m'a peut-être répondu que je n'y avais pas droit parce qu'ils étaient mon papa et ma maman, [...] enfin on m'a sans doute dit quelque chose de ce genre mais ça n'a pas suffi ou je n'en avais pas envie, je veux dire de cette réponse $[\ldots]^{49}$. 
Toute la force du roman, son envoûtement et sa répulsion, réside dans ce refus. Dans une entrevue, Nelly Arcan avait eu cette boutade révélatrice : «Ah! la castration... Tout le mal du monde vient de là, je vous dis! ${ }^{50}$ »

Comme son titre l'indique, le roman Borderline traite aussi de l'excès et des limites. Deux titres de chapitre sur neuf s'intitulent Borderline. Dans les deux extraits tirés du DSM-IV, on souligne l'instabilité des images du soi et des affects. Et c'est bien la tonalité générale du roman. La protagoniste, narratrice au je, y est à la fois triomphatrice et souffrante, victime et tortionnaire. Toute l'ambivalence des sentiments semble condensée dans son nom Sissi Labrèche, qui suscite le rire. Sissi évoque tout autant l'impératrice, la «petite princesse ${ }^{51}$ », que la séductrice ( Suce $\left.^{52} »\right)$ et le double consentement, alors que Labrèche, «mon nom de famille dysfonctionnelle », dira-t-elle, " c'est le trou, c'est la brèche, c'est la fente de mon petit $\operatorname{corps}^{53}$ ». Sissi Labrèche est une « petite souris sans queue ${ }^{54}$ ». Il y a donc un manque radical qui est inscrit dans le nom. Lorsqu'elle se dit spécialiste de la région d' " en bas de la ceinture », image qui renvoie à la fois aux injures et à la sexualité, elle affirme : "Je suis une castratrice. Une cantatrice de la castration $^{55}$ ». J'ajouterais à l'explication onomastique l'aspect hypothétique de la brèche. Qu'arrive-t-il si s'il y a une brèche ? Un vide. Le rien. Le personnage semble tout faire pour éviter de se rendre jusque-là (l'assomption de son identité de brèche, de rien, de solitude radicale).

L'action commence d'ailleurs par une scène de sexe, plutôt explicite, où Sissi, tout en faisant la démonstration du pas-de-rapport sexuel, avoue avoir besoin du regard de l'Autre pour se donner consistance : «Et lui, quand il me regarde avec ses yeux qui roulent comme des billes, il me donne l'impression que je lui suis nécessaire ${ }^{56} »$. Le cynisme fait douter de l'utilité de l'exercice pour le sujet. Le montage de la séduction fonctionne, Sissi se fait l'objet du désir de l'Autre, une «Cendrillon», comme le suppose le titre du premier chapitre, mais il y a un moment limite où elle ne peut plus. La scène de sexe avec Éric se répète au chapitre 9, mais le personnage principal «ferme les cuisses» et finit par fracasser un miroir «en mille $\operatorname{morceaux}^{57} \gg$. Finie l'image du moi idéal, objet d'amour du regard de l'Autre. Beaucoup de parallèles peuvent être faits avec Borderline, l'évocation des contes de fées, l'emprise encore une fois de la séduction pour la femme.

Tout le roman semble construit pour montrer l'inutilité de l'entreprise de séduction et, surtout, le danger de la lignée maternelle. Car, dans Borderline, l'enjeu n'est pas de séduire le père, mais de sortir de la lignée maternelle. Pour Sissi, en quête d'amour et d'attention, cette lignée de femmes s'inscrit dans le désespoir : «Je dois être suicidaire comme ma mère dans trois ans. Ça doit être dans mes gènes, d'être suicidaire. Dans mes gènes remplis d'hérédité malade ${ }^{58}$ ». Même son nom de famille est rattaché à la mort, il a été "laissé, oublié par mon grand-père Labrèche mort d'un cancer du poumon à l'hôpital Notre-Dame ${ }^{59}$ ». La première chose que la grand-mère aurait dite lors du décès de son mari est «Bon débarras ! ${ }^{60} »$.

On sait très peu de chose du père biologique, la grand-mère l'utilise comme une menace ultime : «Si t'es méchante comme ça, je vais appeler ton vrai papa, Papa Méchant ${ }^{61} »$. Si on tient compte du comportement démentiel de la grand-mère à l'égard de celui qui devient son nouveau gendre, on peut douter du bien-fondé de la méchanceté du père. En effet, non contente de le traiter de « ostie de fou » ou de " gros porc ${ }^{62}$ », elle cherche à convaincre la petite Sissi de dénoncer son beau-père comme abuseur ${ }^{63}$. Même si celle-ci le trouve plutôt gentil ${ }^{64}$ et refuse, dans un premier temps, elle finira par accéder aux demandes de la grand-mère. Cette complicité, 
qui correspond, pour elle, à une trahison, puisqu'elle sera la cause, à long terme, de la rupture du couple, est le début de sa croisade : «C'est de ma faute, j'ai vendu ma patrie à l'ennemi pour avoir la paix. Je suis une petite transfuge, une petite traîtresse, une belle petite Judas de cinq ans. Dorénavant, mes jeux n'auront plus de frontières et je serai en guerre contre l'humanité, mais surtout contre moi-même ${ }^{65}$ ».

Que reste-t-il après l'exclusion de la figure paternelle ? Des traits identificatoires qui ont des accents de désespoir. En vain chercherions-nous une image de soi qui se tienne et qui ne vacille pas lorsque le désir du sujet est convoqué dans toute sa foudroyante ardeur. Après une vaine tentative amoureuse avec une partenaire féminine, Saffie, Sissi envisage la mort comme unique voie de sortie. Sans doute la délicieuse ironie ou l'amusant cynisme qui caractérisent la narration empêche l'histoire de se terminer dans la tragédie. Ce sont les niaiseries qui gagnent. À la suite de la grand-mère nous pourrions dire : « Est «bonne en crisse pour raconter des histoires » ou bien encore douter de la validité de cette ensorceleuse narcissique de borderline. Ne dit-elle pas elle-même : «Qu'est-ce que je raconte ? Ce n'est même pas vrai. Je viens juste d'imaginer ça. Je dis tout le temps des niaiseries, calvaire ${ }^{66} »$. La véritable brèche qu'ouvre le récit est l'autodérision, la distance d'avec le discours du moi. Les derniers mots du texte maintiennent la solution du fantasme, que tout le roman semblait mettre en pièce : alors qu'elle vient de rencontrer un " ange sexuel aux ailes cassées" après sa sortie d'hôpital, elle s'avoue : "Ils peuvent porter des chandails de Pantera et oublier de se laver, mais ça n'enlève pas pour autant le charme. Et les yeux fermés, couchée nue dans un lit avec un prince, on ne fait plus la différence ${ }^{67} »$.

En guise de conclusion, je reprendrai une citation de Putain et je ferai mienne son inquiétude : «[...] je pourrais vous décrire la beauté du monde si je savais la voir, raconter comment la foi et le courage peuvent venir à bout des plus grands malheurs $[\ldots]^{68} »$. Où trouverons-nous en effet la foi et le courage de raconter la beauté du monde et de la perpétuer devant l'échec de notre société et de nos gouvernants à représenter le croyable pour soutenir les sujets devant l'horreur du Réel? « [M]ais où va-t-on dans cette société où les filles sont putains et les pères clients ${ }^{69} »$, dira ailleurs Nelly Arcan?

La science d'aujourd'hui, qui prétend parfois remplacer la religion dans l'espace du croyable, n'a plus guère le temps de récupérer le discours féminin de l'excès comme on le faisait autrefois pour les mystiques ou les possédées. Elle offre bien sûr des diagnostics, borderline ou neurasténique, mais, comme le montrent si bien Marie-Sissi Labrèche ou Nelly Arcan, les classifications aident-elles à vivre? À quoi servent les étiquettes quand il s'agit de gérer sa jouissance? Au nom de quoi les jeunes filles doivent-elles ou peuvent-elles renoncer à l'autoréification, à la position d'objet? À la suite de l'analyse de ces deux œuvres, il est troublant de voir que la femme, ce « continent noir », se représente encore sous les traits de la folle ou de la séductrice... Il est vrai que la dénonciation atteint des niveaux inégalés de lucidité et de férocité. Les vérités implacables énoncées dans les œuvres de ces jeunes auteures ne constituentelles pas déjà le dépassement du diagnostic de l'impasse?

\section{BIBLIOGRAPHIE}

APOLLON, Willy, « La rupture de l'expérience freudienne dans la sexualité », Savoir, vol. 5, n ${ }^{\text {os }}$ 1 et 2 septembre 2000, p. 191-222. 
ARCAN, Nelly, Putain, Paris, Seuil, 2001, 187 p.

ARCAN, Nelly, Folle, Paris, Seuil, 2004, 205 p.

BOISCLAIR, Isabelle, «Accession à la subjectivité et autoréification : statut paradoxal de la prostituée dans Putain de Nelly Arcan », L’Écriture du corps dans la littérature québécoise depuis 1980, sous la direction de Daniel Marcheix et Nathalie Watteyne, Limoges, Pulim, 2007, p. 111-123.

COUTURE, François, «Nelly Arcan 3D », site internet des éditions de l'Effet pourpre, [en ligne] [(http://www3.sympatico.ca/effetpourpre/entr-arcan.htm] (17 mai 2007)

HAVERCROFT, Barbara, «Prostitution et autofiction illisible : Putain de Nelly Arcan », publié sur Fabula le 24 octobre 2004 [en 1 ligne]. [http://www.fabula.org/lodel/hebergement_colloques/document42.php] (13 août 2007)

LABRECHE, Marie-Sissi, Borderline, Montréal, Boréal, 2003 [1 ${ }^{\text {re }}$ éd. : 2000], 160 p.

LABRÈCHE, Marie-Sissi, La Brèche, Montréal, Boréal, 2002, 159 p.

LABRÈCHE, Marie-Sissi, La Lune dans un HLM, Montréal, Boréal, 2006, 251 p.

LAROCHELLE, Claudia, «Nelly Arcan, dans l'intimité de la fée du clavier », Canoë.ca, [en ligne]. [ [http://www.canoe.com/divertissement/livres/entrevues/2007/01/27/3466289jdm.html] (13 août 2007)

PRUS, Elena, " "Scandaleusement intime": Putain de Nelly Arcan», [en ligne] [http://st.ulim.md/download/icfi/publicatii/parc_queb/elena_prus208.pdf] (13 août 2007)

PUHL, Andrea, Nelly Arcan : la prostitution et la politique sexuelle dans Putain, mémoire de maîtrise, Université de Saskatchewan, 2005, [en ligne]. [http://library2.usask.ca/theses/submitted/etd-12202005204821/unrestricted/AndreaPuhlThesis1.pdf̃] (13 août 2007)

\section{NOTES}

${ }^{1}$ Willy Apollon, «La rupture de l'expérience freudienne dans la sexualité », Savoir, vol. 5, $\mathrm{n}^{\text {os }} 1$ et 2 septembre 2000, p. 206.

${ }^{2}$ Marie-Sissi Labrèche, La Lune dans un HLM, Montréal, Boréal, 2006, p. 14.

${ }^{3}$ Nelly Arcan, citée par Claudia Larochelle, «Nelly Arcan, dans l'intimité de la fée du clavier », Canoë.ca, [en ligne]. [http://www.canoe.com/divertissement/livres/entrevues/2007/01/27/3466289-jdm.html] (13 août 2007)

${ }_{5}^{4}$ Nelly Arcan, Folle, Paris, Seuil, 2004, p. 18.

${ }_{6}^{5}$ Ibid., p. 52.

${ }^{6}$ La jaquette de l'ouvrage joue bien visuellement avec le parallèle du titre et du nom de l'auteur.

${ }^{7}$ Marie-Sissi Labrèche, La Brèche, Montréal, Boréal, 2002, p. 19.

${ }^{8}$ Ibid., p. 34.

${ }^{9}$ Marie-Sissi Labrèche, Borderline, Montréal, Boréal, 2003 [1 ${ }^{\text {re }}$ éd. : 2000], p. 76. Voir aussi, Marie-Sissi Labrèche, La Brèche, p. 15 et Borderline, p. 16.

${ }^{10}$ Marie-Sissi Labrèche, Borderline, p. 40. Pour un parallèle semblable, voir encore Borderline, p. 15 et La Brèche, p. 16.

${ }^{11}$ Marie-Sissi Labrèche, La Brèche, p. 11.

${ }^{12}$ Ibid., p. 22.

${ }^{13}$ Ibid., p. 15.

${ }^{14}$ Marie-Sissi Labrèche, La Lune dans un HLM, p. 11.

${ }^{15}$ Marie-Sissi Labrèche, Borderline, p. 16.

${ }^{16}$ Marie-Sissi Labrèche, La Brèche, p. 25, 37 et 38. 
${ }^{17}$ Nelly Arcan, Putain, p. 17.

${ }^{18}$ Ibid., p. 19.

${ }^{19}$ Ibid., p. 137.

${ }^{20}$ Ibid., p. 17, 20, 61, 118.

${ }^{21}$ Ibid., p. 54.

${ }^{22}$ Ibid., p. 139 et 146.

${ }^{23}$ Ibid., p. 182.

${ }^{24}$ Ibid., p. 183.

${ }^{25}$ Ibid., p. 65.

${ }^{26}$ Ibid., p. 97 et 143 .

${ }^{27}$ Ibid., p. 103.

${ }^{28}$ Ibid., p. 144.

${ }^{29}$ Voir Andrea Puhl, Nelly Arcan : la prostitution et la politique sexuelle dans Putain, mémoire de maîtrise, Université de Saskatchewan, 2005, [en ligne]. [http://library2.usask.ca/theses/submitted/etd-12202005204821/unrestricted/AndreaPuhlThesis1.pdf̃] (13 août 2007)

${ }^{30}$ Putain, p. 166.

${ }^{31}$ Nelly Arcan, Putain, p. 34.

${ }^{32}$ Ibid., p. 23.

${ }^{33}$ Ibid., p. 57, 16 et 17.

${ }_{35}^{34}$ Ibid., p. 17.

${ }^{35}$ Isabelle Boisclair, « Accession à la subjectivité et autoréification : statut paradoxal de la prostituée dans Putain de Nelly Arcan ", L'Écriture du corps dans la littérature québécoise depuis 1980, sous la direction de Daniel Marcheix et Nathalie Watteyne, Limoges, Pulim, 2007, p. 111-123. Voir aussi Barbara Havercroft, «Prostitution et autofiction illisible: Putain de Nelly Arcan», Fabula, 24 octobre 2004 [en ligne]. [http://www.fabula.org/lodel/hebergement_colloques/document42.php] (13 août 2007)

${ }^{36}$ Ibid., p. 20 et 51.

${ }^{37}$ Ibid., p. 142. Le chiffre trois est symboliquement lourd de sens pour la psychanalyse (importance du tiers dans la relation entre la mère et l'enfant).

${ }^{38}$ Ibid., p. 51.

${ }^{39}$ Ibid., p. 30.

${ }^{40}$ Ibid., p. 92.

${ }^{41}$ Ibid., p. 102.

${ }^{42}$ Ibid., p. 149-150.

${ }^{43}$ Ibid., p. 56.

${ }^{44}$ Ibid., p. 74 ; voir aussi p. 42-43.

${ }^{45}$ Ibid., p. 86.

${ }^{46}$ Ibid., p. 85.

${ }^{47}$ " C'est vrai que je suis injuste » (ibid., p. 49); « ce n'est pas aussi pitoyable que je le prétends » (ibid., p. 58).

${ }^{48}$ Ibid., p. 98.

${ }^{49}$ Ibid., p. 183.

${ }^{50}$ Nelly Arcan, citée par François Couture, «Nelly Arcan 3D », site internet des éditions de l'Effet pourpre, [en ligne] [(http://www3.sympatico.ca/effetpourpre/entr-arcan.htm] (17 mai 2007)

${ }_{51}^{51}$ Marie-Sissi Labrèche, Borderline, p. 59.

${ }^{52}$ Ibid., p. 44 et 63.

${ }^{53}$ Ibid., p. 63.

${ }_{55}^{54}$ Ibid., p. 61.

${ }_{55}^{55}$ Ibid., p. 50.

${ }_{56}^{56}$ Ibid., p. 16.

${ }_{58}^{57}$ Ibid., p. 150-151.

${ }^{58}$ Ibid., p. 61.

${ }^{59}$ Ibid., p. 63.

${ }^{60}$ Ibid., p. 131-132. 
${ }^{61}$ Ibid., p. 123.

${ }^{62}$ Ibid., p. 122.

${ }^{63}$ Ibid., p. 130.

${ }^{64}$ Ibid., p. 131

${ }^{65}$ Ibid., p. 135.

${ }^{66}$ Ibid., p. 20.

${ }_{68}^{67}$ Ibid., p. 158.

${ }^{68}$ Nelly Arcan, Putain, p. 79-80.

${ }^{69}$ Ibid., p. 50-51. 